\title{
Thymic and bone marrow output in individuals with 22q11.2 deletion syndrome
}

\author{
Nina Darr ${ }^{1-3}$, Doron Gothelf ${ }^{2,3}$, David Korn', Amos Frisch ${ }^{4}$, Abraham Weizmann ${ }^{4,5}$, Elena Michaelovsky4, \\ Miri Carmel ${ }^{4}$, Yonatan Yeshayahu ${ }^{1,3,6}$, Gal Dubnov-Raz ${ }^{1,3}$, Itai M. Pessach ${ }^{3,7}$, Amos J. Simon' ${ }^{1}$, Atar Lev' and Raz Somech ${ }^{1,3}$
}

BACKGROUND: The 22q11.2 deletion syndrome (22q11.2DS) is a congenital multisystem anomaly characterized by typical facial features, palatal anomalies, congenital heart defects, hypocalcemia, immunodeficiency, and cognitive and neuropsychiatric symptoms. The aim of our study was to investigate T- and B-lymphocyte characteristics associated with 22q11.2DS.

METHODS: Seventy-five individuals with 22q11.2DS were tested for T and B lymphocytes by examination of T-cell receptor rearrangement excision circles (TRECS) and B-cell k-deleting recombination excision circles (KRECS), respectively.

RESULTS: The 22q11.2DS individuals displayed low levels of TRECs, while exhibiting normal levels of KRECs. There was a significant positive correlation between TREC and KREC in the 22q11.2DS group, but not in controls. Both TREC and KREC levels showed a significant decrease with age and only TREC was low in 22q11.2DS individuals with recurrent infections. No difference in TREC levels was found between 22q11.2DS individuals who underwent heart surgery (with or without thymectomy) and those who did not.

CONCLUSION: T-cell immunodeficiency in 22q11.2DS includes low TREC levels, which may contribute to recurrent infections in individuals with this syndrome. A correlation between T- and B-cell abnormalities in 22q11.2DS was identified. The B-cell abnormalities could account for part of the immunological deficiency seen in 22q11.2DS.

$\mathbf{T}$ he 22q11.2 deletion syndrome (22q11.2DS) is a multisystem congenital disorder caused by a microdeletion of one of the two copies of chromosome 22q11.2 (1-3). It occurs in $\sim 1 / 4,000$ live births and is characterized by typical facial features, palatal anomalies, congenital heart defects, hypocalcemia, immune deficits, intellectual disability and neuropsychiatric symptoms (4). The immunodeficiency in 22q11.2DS, which varies in its severity, appears in $65-77 \%$ of patients and leads to increased susceptibility to recurrent infections and higher rate of autoimmune disorders (5). While the major immunodeficiency in
22q11.2DS involves the cellular arm of the immune system (6), some humoral abnormalities have been described $(7,8)$. The majority of 22q11.2DS individuals exhibit mild-to-moderate reductions in the number of $\mathrm{T}$ cells and only a mild deficit in T-cell function (6-9). The T-cell immunodeficiency is due to abnormal thymic migration in most cases and not necessarily due to a full thymic aplasia. Thus, residual microscopic nests of thymic epithelial cells account for some ability to produce T cells (8). Nevertheless, in $\sim 1 \%$ of patients, there is a complete absence of thymus ("complete" DiGeorge syndrome), causing severe T-cell immunodeficiency, mimicking severe combined immunodeficiency (SCID) phenotype $(5,10)$, and should be diagnosed early in life. These patients might benefit from hematopoietic stem cell transplantation (HSCT). In the absence of a functional thymus, it appears that the immune reconstitution by HSCT is achieved through peripheral expansion of mature donor T cells, brought in by the graft (11). Thymic transplantation has also been shown to restore normal immune function with the generation of naive thymic emigrants (12). Another major manifestation in 22q11.2DS is congenital heart defects (13). These may require heart surgeries that often result in thymectomy, further hindering the already deficient immune system of these patients $(14,15)$. A better characterization of the immunodeficiency in 22q11.2DS will improve our understanding of the long-term health prognosis and quality of life in these patients. Moreover, since the syndrome is ultimately diagnosed using fluorescence in situ hybridization (FISH), which is costly, in many cases the diagnosis is based only on clinical assessment, which often leads to misdiagnosis (16). Due to the high occurrence of immunological deficits in individuals with 22q11.2DS, using a diagnostic method relying on immunological characteristics may be helpful. More importantly, such an immunologically based diagnosis is crucial and can be lifesaving early in life for individuals with "complete" DiGeorge syndrome. One of the ways to assess immunological deficits is the analysis of T- and B-cell receptors (TCRs and BCRs, respectively). Functional receptors are created through the ligation of coding joint $(\mathrm{cj})$ recombination

\footnotetext{
'Pediatric Department B and Immunology Services, Jeffrey Modell Foundation Center, Sheba Medical Center, Tel Hashomer, Israel; ${ }^{2}$ The Behavioral Neurogenetics Center, Sheba Medical Center, Tel Hashomer, Israel; ${ }^{3}$ Edmond and Lily Safra Children's Hospital, Sheba Medical Center, Tel Hashomer, Affiliated to the Sackler Faculty of Medicine, Tel Aviv

University, Tel Aviv, Israel; ${ }^{4}$ Felsenstein Medical Research Center, Petah Tikva, Israel; ${ }^{5}$ Geha Mental Health Center, Petah Tikva, Israel; ${ }^{6}$ Pediatric Endocrinology Unit, Sheba Medical Center, Tel Hashomer, Israel; ${ }^{7}$ Department of Pediatric Critical Care, Sheba Medical Center, Tel Hashomer, Israel. Correspondence: Raz Somech (raz.somech@sheba.health.gov.il) 


\section{Articles | Daret al.}

sites. The byproducts of these processes, namely T-cell receptor rearrangement excision circles (TRECs) and $\kappa$-deleting recombination excision circles (KRECs), are formed through signal joint (sj) recombination sites. sj-TRECs and sj-KRECs are hence DNA markers of the thymic and B-lymphocyte bone marrow output, respectively, since they are present exclusively in naive $\mathrm{T}$ and $\mathrm{B}$ cells that have recently emigrated from these organs (17). sj-TRECs have previously been shown to be very low in individuals with 22q11.2DS (6). Moreover, sj-TREC levels have been found to decrease with age both in healthy individuals and in patients with 22q11.2DS. Interestingly, in normal individuals, a faster decrease was seen in men compared with women (18). KREC has also been examined in normal individuals and was found to be constant with age and among different genders (18). Pioneering screening of newborns for SCID using TREC had already been performed by Kwan et al. (19) and Routes et al. (20) and was found to be successful in the early diagnosis of SCID patients including some cases of "complete" DiGeorge syndrome. Some individuals with 22q11.2DS were found to have low TREC levels later in infancy. One possible explanation for this observation is the removal of the thymus gland during cardiac operation. $49-83 \%$ of individuals with 22q11.2DS have cardiac abnormalities $(13,21)$ that require early surgical intervention and
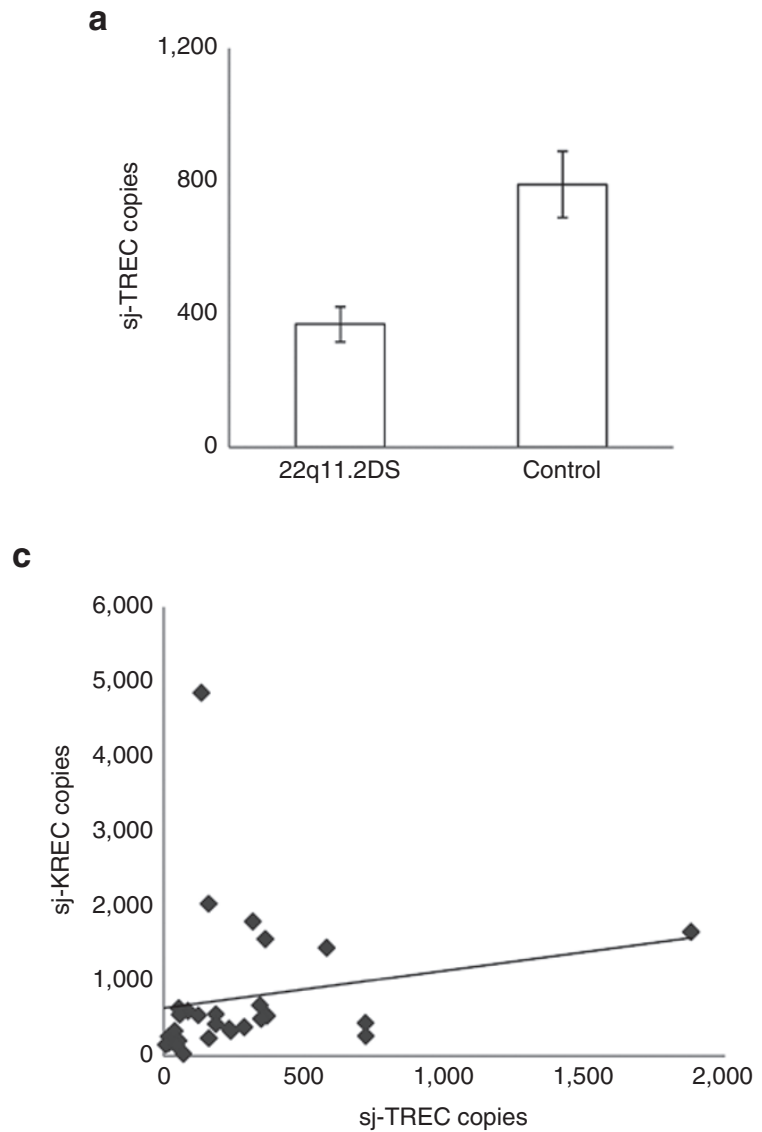

removal of the adjacent thymus gland, therefore finding low TREC levels in these patients is very likely $(14,15)$.

The aim of this study was to evaluate sj-TREC and sj-KREC levels in the peripheral blood of individuals with 22q11.2DS, specifically with regard to the performance of heart surgery, in order to better evaluate the immunological characteristics associated with the syndrome.

\section{RESULTS}

\section{Clinical Findings in Individuals with 22q11.2DS}

Data were available for 75 individuals with 22q11.2DS (median age $15 \mathrm{y}$, interquartile range 8-24 y; 45 males) and 36 healthy controls in which immunodeficiency was clinically excluded (median age $14 \mathrm{y}$, interquartile range $12-15 \mathrm{y} ; 24$ males). There was no significant difference in age and sex distribution between the groups ( $P=0.541$ and 0.537 , respectively).

The main presenting symptom in individuals with 22q11.2DS was abnormal facies, which appeared in 56 patients (75\%). Palatal anomalies were noted in 56 patients as well (75\%) and 31 of them underwent pharyngeal flap surgery (41\%). Cardiac abnormalities were found in 44 patients (59\%), 24 of whom required surgery. Twenty-three patients $(31 \%)$ had hypocalcemia, and nine patients (12\%) had lymphopenia. Other nonclassical symptoms were autoimmune diseases such as psoriasis,
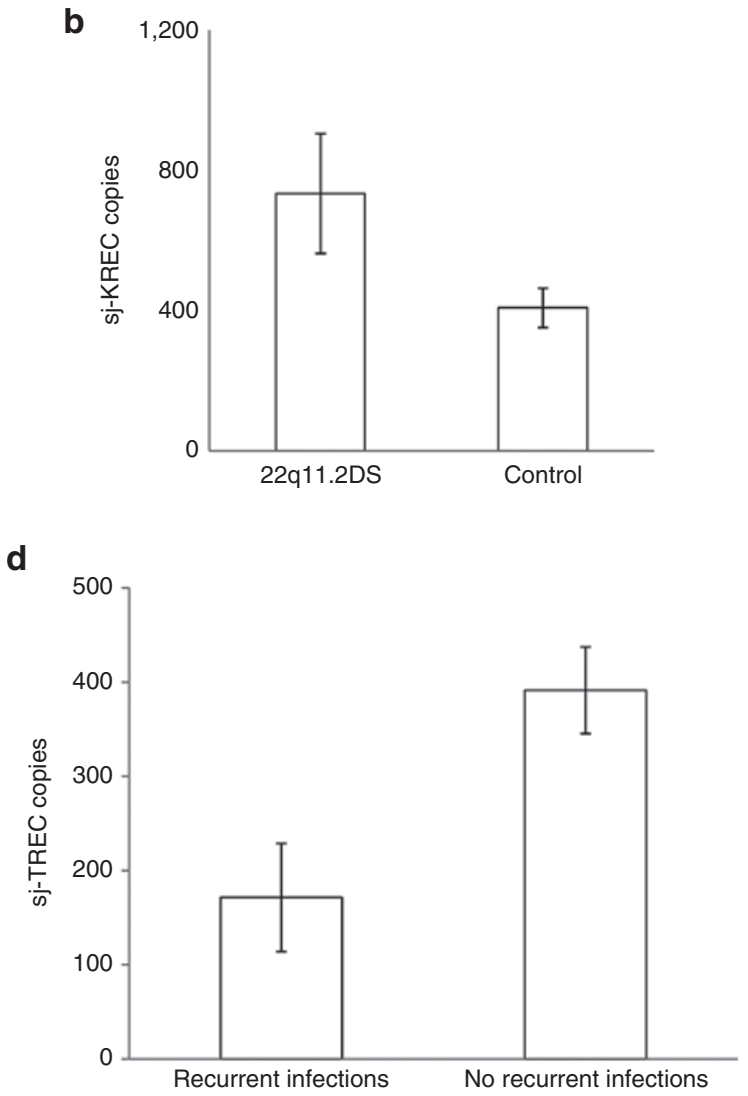

Figure 1. sj-TREC and sj-KREC copies in 22q11.2DS patients. sj-TREC (a) and sj-KREC (b) copies of 22q11.2DS patients $(n=73$ and $n=31$, respectively) are shown compared with age-matched healthy controls $(n=36)$. A correlation between sj-KREC and sj-TREC is presented for 22q11.2DS patients $(\mathbf{c}, n=31)$. sj-TREC (d) copies of 22q11.2DS patients with recurrent infections $(n=7)$ are shown compared with 22q11.2DS patients without recurrent infections $(n=66)$. 
vitiligo, and glomerulonephritis (11 patients, 15\%), severe recurrent infections ( 7 patients, $10 \%$ ), mild thrombocytopenia defined as platelet counts $50-100 \times 10^{3}$ (14 patients, $20 \%$ ), significant thrombocytopenia defined as platelet counts $<50 \times 10^{3}$ ( 5 patients, $7 \%$ ), anemia (5 patients, $7 \%$ ), hypothyroidism (5 patients, $7 \%$ ), and obesity (10 patients, 13\%). The mean full-scale intelligence quotient (IQ) of the patient group was 77.1 \pm 13.7 .

\section{Characterization of TREC and KREC in Patients Compared with Controls and Correlation with Recurrent Infections}

Individuals with 22q11.2DS displayed lower levels of TREC $(P=0.0004)$, while exhibiting a nonsignificant difference in the levels of KREC $(P=0.07)$ compared with healthy individuals (Figure 1a,b, respectively). The levels of TREC and KREC mildly correlated with each other when examined for both the 22q11.2DS and control groups together, with borderline statistical significance $\left(R_{\mathrm{s}}=0.23, P=0.057\right)$. However, when examined for individuals with 22q11.2DS only, a more significant correlation was found (Figure $1 \mathrm{c} ; R_{\mathrm{s}}=0.47, P=0.01$ ). Both TREC and KREC levels did not correlate with the lymphocyte numbers $\left(R_{\mathrm{s}}=0.34, P=0.066 ; R_{\mathrm{s}}=0.1, P=0.74\right.$, respectively).
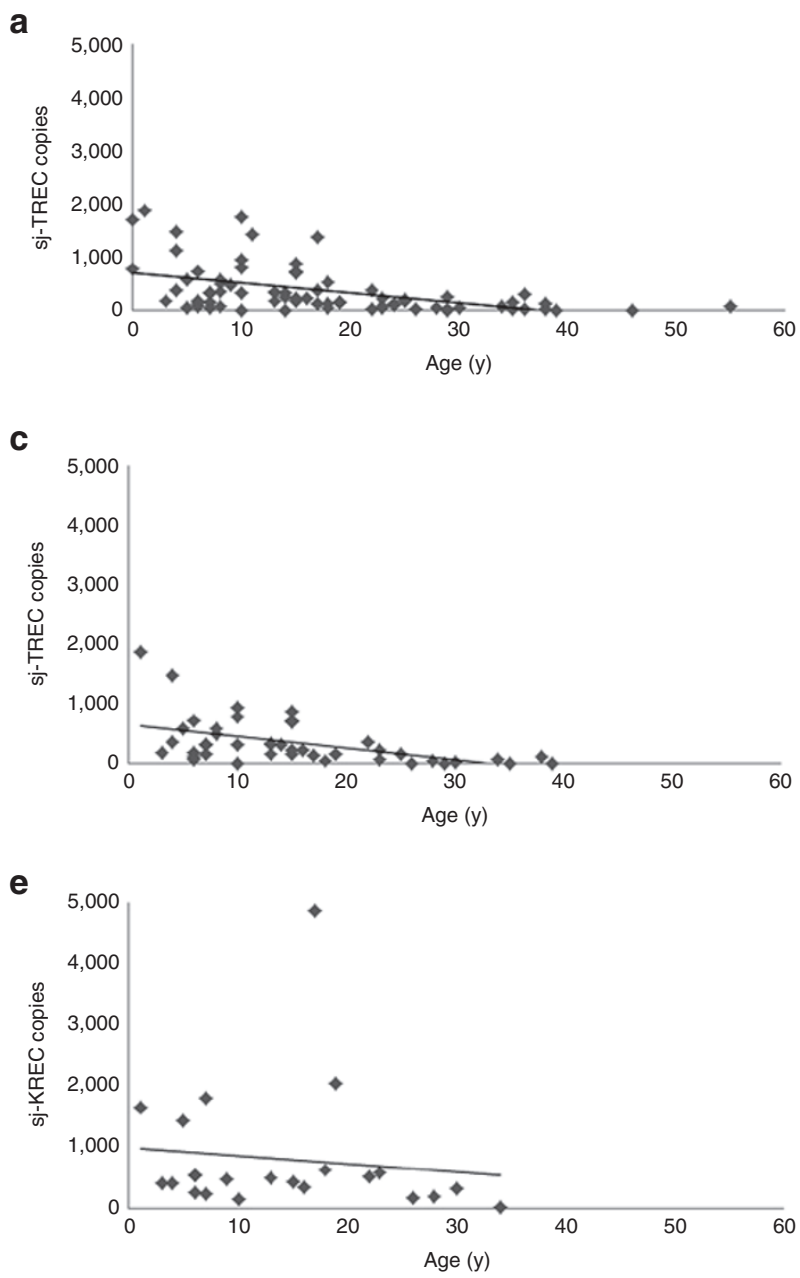

Information about recurrent infections was available for 73 individuals with 22q11.2DS. $10 \%$ of them (7 patients) were classified as having recurrent infections. Their mean age was similar to that of individuals without recurrent infections $(P=0.6)$. This group of patients displayed lower TREC levels compared with patients without recurrent infections (Figure 1d, $P=0.005$ ), yet one patient in this group had TREC values that deviate 9 SDs from the average and therefore was taken out from the group. The relation between KREC levels and recurrent infections could not really be established as there were only two participants who have a KREC result and had infections.

TREC and KREC Levels in 22q11.2DS Patients Decrease with Age Both TREC and KREC levels of individuals with 22q11.2DS showed a significant decrease with age (Figure $2 \mathrm{a}, R_{\mathrm{s}}=-0.57$, $P<0.001$; Figure $2 \mathrm{~b}, R_{\mathrm{s}}=-0.42, P=0.01$, respectively). When examined separately for each sex, TREC levels revealed significant and similar correlations with age for both males and females (Figure 2c; $R_{\mathrm{s}}=-0.58, P=0.000$; Figure 2d; $R_{\mathrm{s}}=-0.52$, $P=0.003$, respectively). The separate correlations for males
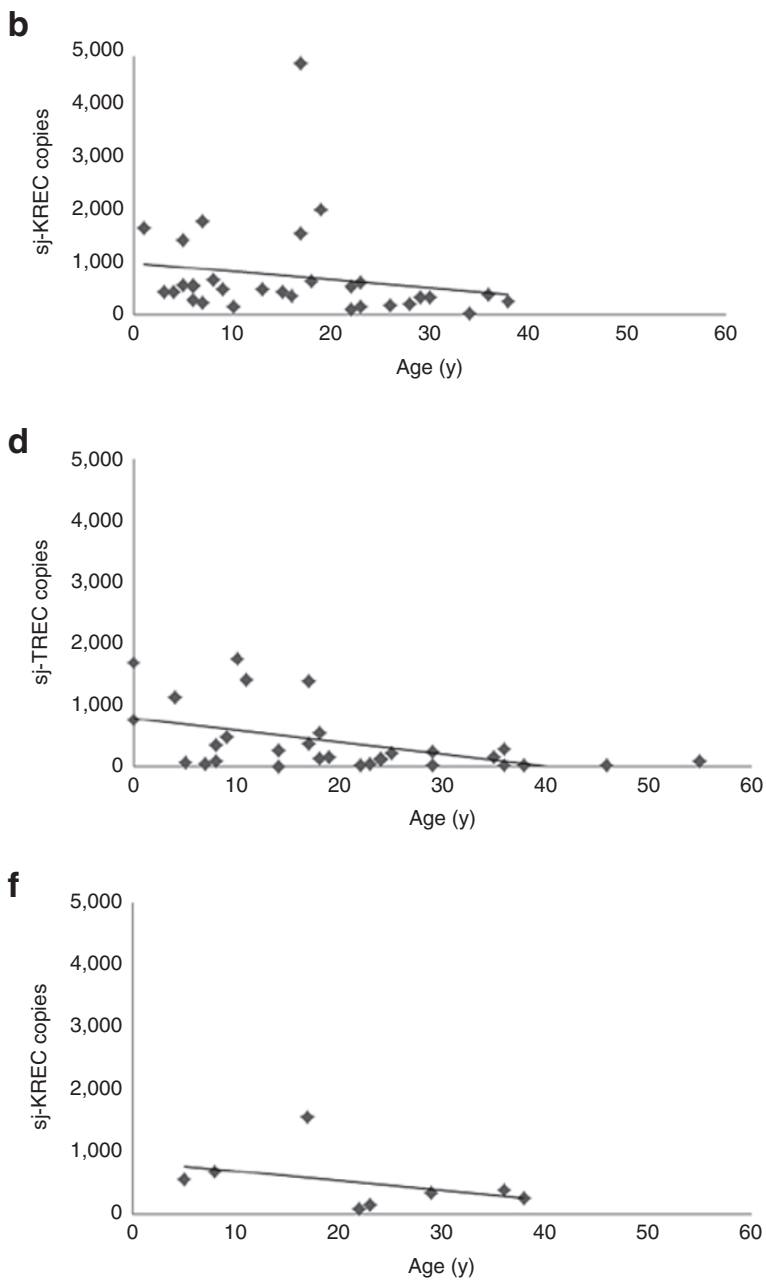

Figure 2. sj-TREC and sj-KREC correlations with age in 22q11.2DS patients. sj-TREC (a) and sj-KREC (b) correlations with age, for 22q11.2DS patients, are presented. Further, correlations are shown separately for males: sj-TREC (c, $n=43)$, sj-KREC (e, $n=23)$, and females: sj-TREC (d, $n=30)$, sj-KREC (f, $n=8)$. 
a

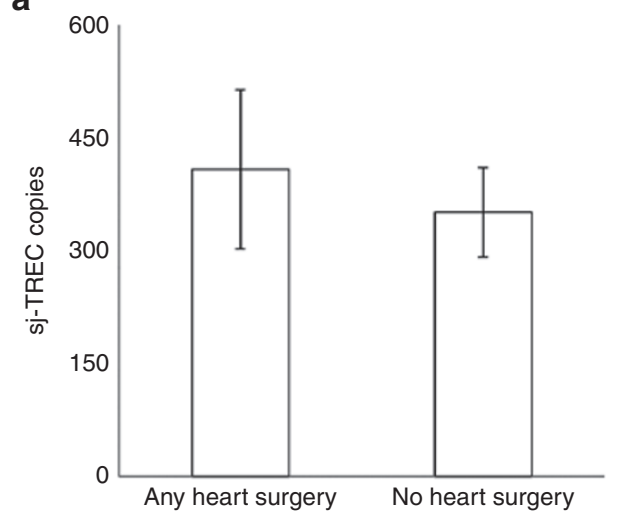

b

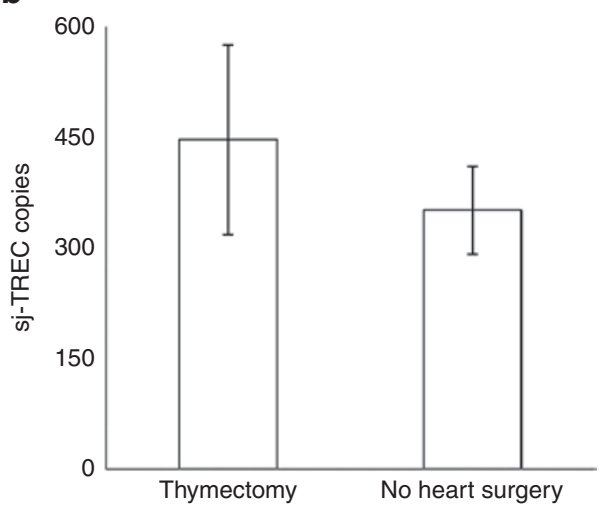

Figure 3. sj-TREC levels of 22q11.2DS patients who went through heart surgeries compared with those who did not. sj-TREC copies of patients who went through any heart surgery $(n=24)$ are compared with 22q11.2DS patients who did not go through heart surgeries at all $(n=49)$ (a). Of those who went through heart surgeries, only the patients who went through surgeries that required thymectomy (ASD, VSD, TOF, and valve replacement) were chosen and their sj-TREC values $(n=19)$ are compared with patients who did not go through heart surgeries at all $(n=49)(\mathbf{b})$.

and females of KREC vs. age were not significant (Figure 2e; $R_{\mathrm{s}}=-0.26, P=0.21$; Figure 2f; $R_{\mathrm{s}}=-0.5, P=0.2$, respectively).

\section{Low TREC Levels Are Not Related to Heart Surgeries and Thymectomy}

TREC levels of individuals with 22q11.2DS who underwent any type of cardiac surgery (i.e., repair of atrial septal defect (ASD), ventricular septal defect (VSD), teratology of fallot (TOF), valve replacement, or blood vessel-related surgeries) were compared with TREC levels of patients who did not undergo such surgery. No significant difference in TREC levels was found between these subgroups (Figure 3a; $P=0.61$ ). Next, only the patients who underwent cardiac surgeries requiring midsternotomy and thus thymectomy were examined (ASD, VSD, TOF, and valve replacement). More specifically, no difference in TREC levels was found between patients who underwent cardiac surgeries requiring midsternotomy and thus thymectomy and the patients who did not undergo such surgery (Figure $3 \mathbf{b} ; P=0.44$ ).

\section{DISCUSSION}

The aim of this study was to evaluate the TREC and KREC levels in patients with 22q11.2DS, in order to better understand the immunological characteristics associated with this syndrome. Seventy-five patients with 22q11.2DS were evaluated in this study and compared with healthy controls. The clinical features of the 22q11.2DS patients were similar to those previously described by other groups (1-10).

A main characteristic of 22q11.2DS is immunodeficiency, which is also responsible for major adverse health effects of this syndrome. It is important to characterize and understand this aspect of 22q11.2DS as best as possible, in order to improve patient prognosis and care. Indeed, various immunological assays are widely used in these patients to determine the status of their immunity. These include lymphocyte enumeration (by fluorescence activated cell sorting (FACS) analysis) and lymphocyte function (by assessment of the response to mitogenic stimulations). Yet, these tests do not reflect the actual thymic activity, are expensive and elaborative requiring fresh blood samples, and thus are with limited added-value to accurately assess the immune system of these patients. The introduction of a novel molecular methodology, e.g., TREC quantification, as a reliable and sensitive tool to assess thymic function is extremely important in 22q11.2DS patients because of thymic involvement in the disease pathogenesis. Thus, the results obtained from the TREC assay may be used to classify patients based on the status of their immunity, predicting susceptibility to infections. In this study, we found that the TREC levels were significantly lower in individuals with 22q11.2DS compared with healthy controls and tended to decrease with age due to the natural thymic involution, as has been previously shown (6,22-24). Furthermore, lower TREC levels were found in 22q11.2DS patients with recurrent infections, regardless of their age, which probably contributes to the immunodeficiency in some patients.

A novel finding in our study was the similar decline in TREC levels with age in individuals with 22q11.2DS of both sexes. In previous studies, males showed a significant decrease in TREC with age, while females exhibited only a mild reduction (18). This finding further emphasizes the large variability in the immunodeficiency brought about by the deletion in chromosome 22, and the need for individualized and functional assessment of patients in all ages and both sexes.

Even though the main investigated and substantial immunodeficiency seen in 22q11.2DS relates to T-cell defects $(6,25)$, previous findings indicate humoral involvement as well $(7,13)$. For example, in a recent study, 6\% of 22q11.2DS patients over the age of 3 y had hypogammaglobulinemia (12), and in another study peripheral CD27+ B cells were reduced in patients compared with age-matched healthy controls (26). We examined KREC values in 22q11.2DS and found that there was no difference between the KREC levels of patients and those of control participants. However, there was a significant correlation between the TREC and KREC levels in 22q11.2DS, which was not found in the control group. This finding suggests that B cells are also affected in 22q11.2DS, probably in 
the context of severe T-cell abnormalities. This assumption relies on previous studies and the high importance that $\mathrm{T}$ cells have in the activation and regulation of B cells. For example, $\mathrm{T}$ helper cells assist in the maturation of $\mathrm{B}$ cells into plasma cells and memory B cells $(8,27)$. An additional evidence for the involvement of B cells and for the importance of T-B cell interaction is the decrease in patients' KREC values with age, a novel finding that was evident in our study. The importance of this finding is especially pronounced, since previous findings in healthy participants showed that KRECs remain steady throughout life. Therefore, the combination of T- and B-cell deficits, which worsen with age, may explain the increased susceptibility of 22q11.2DS patients to recurrent infections and autoimmune diseases (8), and should be taken into account when composing a treatment for patients with these conditions. Nevertheless, other aspects regarding the B-cell deficiency in 22q11.2DS remain unknown, and should be further investigated.

The gold standard for the detection of 22q11.2DS is FISH, which is costly and time-consuming $(2-3 \mathrm{~d})$. Therefore, a large-scale screening procedure using this technique to identify children with 22q11.2DS as early as possible is problematic and is not being routinely performed. This is one of the main reasons why many children with 22q11.2DS remain undiagnosed. Since one of the major manifestations of 22q11.2DS is congenital heart defects and since as much as $1 \%$ of all children with congenital heart defects are diagnosed with 22q11.2DS (28), one way to improve 22q11.2DS diagnosis is to routinely screen all children with congenital heart defects for 22q11.2DS. However, even in this scenario, a FISH analysis will still be too expensive, and therefore less-expensive methods are still needed and are being examined (29). This need is even more critical when it comes to "complete" DiGeorge syndrome patients with severe immunodeficiency, who require immediate therapeutic interventions, and early diagnosis can be lifesaving. Our results, together with the recent attempts to screen newborns for immunodeficiency $(19,20)$, suggest that TREC might be an appropriate method to achieve this goal. Indeed, in New York State, 18 cases of 22q11.2DS were detected through newborn screening using the TREC assay (30). This assay identified approximately $14.8 \%$ of individuals with the 22q11.2DS but importantly those with severe immune involvement who required immediate medical management. In California, nine patients with 22q11.2DS were identified through TREC newborn screening, where one of these patients received thymus transplant as he presented with "complete" DiGeorge phenotype (19). Recently, simultaneous TREC and KREC analyses in 22q11.2DS patients were performed on their stored original Guthrie cards (31). While all 22q11.2DS patients showed KREC copies within the normal range, a high proportion of the 22q11.2DS patients were identified by the TREC-based screening. These results are similar to our observations. Unlike the healthy population, individuals with 22q11.2DS present very low levels of TREC initially, which decrease even further with age, regardless of sex. This finding may help early in life in pinpointing those subjects who are suspected to carry a deletion in chromosome 22. Our additional novel results that show low TREC levels in all individuals with 22q11.2DS, regardless of whether they underwent heart surgery with or without thymectomy, indicated that low TREC levels are an intrinsic characteristic of the syndrome, not influenced by extrinsic surgical interventions related to congenital heart defects. This further supports the notion of performing screening TREC analysis for all children with congenital heart defects, proceeding then to FISH analyses only with those who exhibit low TREC levels. This approach has a functional aspect, is less costly, and can highly increase our ability to identify hidden cases of 22q11.2DS.

We acknowledge that our study is subject to several limitations inherent to retrospective studies of this nature. We relied on available clinical, surgical reports and administrative data to assess illness severity at the patient or hospital level. Thus, residual confounding cannot be ruled out. We tried at least to eliminate aging, a well-known factor involved in immune reduction even in normal individuals, from our results. In addition, our sample size was limited, and it is possible that an association still exists between some of the examined markers, e.g., abnormal KREC levels and autoimmunity in 22q11.2DS. Lastly, we should confirm that the spectrum of clinical symptoms associated with 22q11.2DS is extremely wide; therefore our results cannot necessarily be generalized to all patients with this syndrome. The B-cell abnormalities could account for some of the immunological diseases of 22q11.2DS. Given the complexity of the immune system, and the broad range of immunodeficiency seen in patients with 22q11.2DS, additional studies should be performed on this topic specifically directed to fill the knowledge gap in B-cell production, maturation, and activity in this special population. In addition, TREC and KREC profiles can serve as indicators for performing a further diagnosis of severe cases of 22q11.2DS. An implementation of newborn screening using TREC assay will enable the identification of some patients with 22q11.2DS. This is highly important for those 22q11.2DS patients who require immediate medical intervention to restore their immune system.

In summary, the aim of this study was to evaluate TREC and KREC levels in patients with 22q11.2DS, as markers of immune system activity. In comparison to what already has been published, we show in this study that: (i) while the T-cell deficiency is a known salient feature in 22q11.2DS, low TREC levels are correlated with recurrent infections; (ii) TREC levels decline with age regardless of patients' gender; (iii) reduced TREC and KREC levels are correlated, suggesting an association between T- and B-cell abnormalities in 22q11.2DS; (iv) KREC values in patients decrease with age, thus may contribute to the development of autoimmunity seen in adult patients; and (v) low TREC levels are detected in patients, regardless of the type of heart surgery they underwent (involving or not involving thymectomy). Taken together, we propose that TREC and KREC enumerations should be an integral part of the immune evaluation of 22q11.2DS patients at any age, especially those with recurrent infections or 
autoimmunity. This may dictate further supportive care anticipatory guidelines, such as avoidance of public places, limited contact with young children, strict hand washing, avoidance of live vaccines, and leukodepletion and irradiation of essential blood products, for some individuals with 22q11.2DS. Furthermore, in young infants with extremely low or even undetectable TREC levels, they should be monitored and treated very cautiously including considering HSCT or thymic transplantation.

\section{METHODS}

\section{Participants}

Seventy-five individuals diagnosed with 22q11.2DS were enrolled to the study from the Behavioral Neurogenetics Center at The Edmond and Lily Safra Children's Hospital, Sheba Medical Center, Tel Hashomer, Israel. The study was approved by the Institutional Review Board, Sheba Medical Center, Tel Hashomer, Israel, and conducted according to the Declaration of Helsinki. The adult participants, or a parent of the pediatric participants, signed an informed consent form. The diagnosis of 22q11.2DS was confirmed in all patients using FISH or multiplex ligation-dependent probe amplification, as previously described (16). The medical records of the 22q11.2DS individuals were reviewed for relevant clinical information. Participants' parents were interviewed for developmental and medical information and the IQ of the 22q11.2DS patients was measured using the age-appropriate Wechsler Intelligence Test as previously described $(32,33)$. Recurrent infections in patients were defined as two or more infections per year who were documented in medical records. Thirty-six healthy individuals were used as controls. All control participants were previously healthy, and had no acute illness during the time of blood drawing. They were sampled during the year 2010-2011 as "healthy controls" for various immunological studies in our laboratory, and were deemed to be immunocompetent. A blood sample was collected from all participants and the DNA was extracted by a commercial kit (Roche Diagnostics, Mannheim, Germany).

\section{Quantification of TRECS and KRECs}

The amount of TREC was determined by real-time quantitative polymerase chain reaction (RQ-PCR). Reactions were performed using $0.5 \mu \mathrm{g}$ genomic DNA extracted from the patients' peripheral blood mononuclear cells. PCRs contained TaqMan universal PCR master mix (Applied Biosystems, Foster City, CA), specific primers $(900 \mathrm{nmol} / \mathrm{l})$, and FAM-TAMRA probes $(250 \mathrm{nmol} / \mathrm{l})$, as previously described $(34,35)$. The RQ-PCR was carried out in an ABI PRISM 7900 Sequence Detector System (Applied Biosystems). The standard curve was constructed by using serial dilutions of a known TREC plasmid (generously provided by Dr Daniel Douek, Vaccine Research Center, National Institute of Allergy and Infectious Diseases, Bethesda, MD). The dilutions contained $10^{3}-10^{6}$ copies of the sj-TREC plasmid. The number of TRECs in a given sample was automatically calculated by comparing the obtained $C_{t}$ value of a patient's sample with the standard curve using an absolute quantification algorithm. The amount of signal and coding joint of KRECs was determined by RQ-PCR as described above for TRECs. The amplification of RNAseP (TaqMan assay, Applied Biosystems) served as a quality control to verify similar amounts of genomic DNA that were used in the assays for both the TREC and KREC analyses. Each experiment was performed in triplicate, and the threshold for $C_{t}$ determination was positioned at the same level of 0.2 each time.

\section{Statistical Analysis}

Continuous variables with more than 10 participants in each group were compared using independent sample $t$-tests. Otherwise, the nonparametric Mann-Whitney $U$-test was used. Correlations between the continuous variables were evaluated using the Spearman's rho test. The data were statistically analyzed by using IBM SPSS statistics version 21 (IBM, Armonk, NY). A $P$ value $<0.05$ was considered significant.

\section{STATEMENT OF FINANCIAL SUPPORT}

The Basil O'Connor Starter Scholar Research Award by the March of Dimes, White Plains, NY, to Gothelf (grant 5-FY06-590); the Binational Science Foundation, Jerusalem, Israel, to Gothelf (grant 2011378).

Disclosure: The authors declare that they have no conflict of interest.

\section{REFERENCES}

1. Burn J. Closing time for CATCH22. J Med Genet 1999;36:737-8.

2. Kirkpatrick JA Jr, DiGeorge AM. Congenital absence of the thymus. Am J Roentgenol Radium Ther Nucl Med 1968;103:32-7.

3. Driscoll DA, Spinner NB, Budarf ML, et al. Deletions and microdeletions of 22q11.2 in velo-cardio-facial syndrome. Am J Med Genet 1992;44:261-8.

4. Motzkin B, Marion R, Goldberg R, Shprintzen R, Saenger P. Variable phenotypes in velocardiofacial syndrome with chromosomal deletion. J Pediatr 1993;123:406-10.

5. Gennery AR. Immunological aspects of 22q11.2 deletion syndrome. Cell Mol Life Sci 2012;69:17-27.

6. Piliero LM, Sanford AN, McDonald-McGinn DM, Zackai EH, Sullivan KE. T-cell homeostasis in humans with thymic hypoplasia due to chromosome 22q11.2 deletion syndrome. Blood 2004;103:1020-5.

7. Junker AK, Driscoll DA. Humoral immunity in DiGeorge syndrome. J Pediatr 1995;127:231-7.

8. McLean-Tooke A, Spickett GP, Gennery AR. Immunodeficiency and autoimmunity in 22q11.2 deletion syndrome. Scand J Immunol 2007;66: $1-7$.

9. Patel K, Akhter J, Kobrynski L, et al. Immunoglobulin deficiencies: the B-lymphocyte side of DiGeorge Syndrome. J Pediatr 2012;161:950-3.

10. Markert ML, Hummell DS, Rosenblatt HM, et al. Complete DiGeorge syndrome: persistence of profound immunodeficiency. J Pediatr 1998;132:15-21.

11. Bensoussan D, Le Deist F, Latger-Cannard V, et al. T-cell immune constitution after peripheral blood mononuclear cell transplantation in complete DiGeorge syndrome. Br J Haematol 2002;117:899-906.

12. Sedivá A, Bartunková J, Zachová R, et al. Early development of immunity in diGeorge syndrome. Med Sci Monit 2005;11:CR182-7.

13. Friedman MA, Miletta N, Roe C, et al. Cleft palate, retrognathia and congenital heart disease in velo-cardio-facial syndrome: a phenotype correlation study. Int J Pediatr Otorhinolaryngol 2011;75:1167-72.

14. Halnon NJ, Cooper P, Chen DY, Boechat MI, Uittenbogaart CH. Immune dysregulation after cardiothoracic surgery and incidental thymectomy: maintenance of regulatory T cells despite impaired thymopoiesis. Clin Dev Immunol 2011;2011:915864.

15. Kurobe H, Tominaga T, Sugano M, et al. Complete but not partial thymectomy in early infancy reduces T-cell-mediated immune response: threeyear tracing study after pediatric cardiac surgery. J Thorac Cardiovasc Surg 2013;145:656-62, 662.e1-2; discussion 662.

16. Michaelovsky E, Frisch A, Carmel M, et al. Genotype-phenotype correlation in 22q11.2 deletion syndrome. BMC Med Genet 2012;13:122.

17. Mensen A, Ochs C, Stroux A, et al. Utilization of TREC and KREC quantification for the monitoring of early T- and B-cell neogenesis in adult patients after allogeneic hematopoietic stem cell transplantation. J Transl Med 2013;11:188.

18. Serana F, Airò $\mathrm{P}$, Chiarini $\mathrm{M}$, et al. Thymic and bone marrow output in patients with common variable immunodeficiency. J Clin Immunol 2011;31:540-9.

19. Kwan A, Church JA, Cowan MJ, et al. Newborn screening for severe combined immunodeficiency and T-cell lymphopenia in California: results of the first 2 years. J Allergy Clin Immunol 2013;132:140-50.

20. Routes JM, Grossman WJ, Verbsky J, et al. Statewide newborn screening for severe T-cell lymphopenia. JAMA 2009;302:2465-70.

21. Guo T, McDonald-McGinn D, Blonska A, et al. Genotype and cardiovascular phenotype correlations with TBX1 in 1,022 velo-cardio-facial/ DiGeorge/22q11.2 deletion syndrome patients. Hum Mutat 2011;32:1278-89.

22. Dion ML, Sékaly RP, Cheynier R. Estimating thymic function through quantification of T-cell receptor excision circles. Methods Mol Biol 2007;380:197-213. 
23. Douek DC, Vescio RA, Betts MR, et al. Assessment of thymic output in adults after haematopoietic stem-cell transplantation and prediction of T-cell reconstitution. Lancet 2000;355:1875-81.

24. Serana F, Chiarini M, Zanotti C, et al. Use of V(D)J recombination excision circles to identify $\mathrm{T}$ - and B-cell defects and to monitor the treatment in primary and acquired immunodeficiencies. J Transl Med 2013;11:119.

25. Chinen J, Rosenblatt HM, Smith EO, Shearer WT, Noroski LM. Long-term assessment of T-cell populations in DiGeorge syndrome. J Allergy Clin Immunol 2003;111:573-9.

26. Finocchi A, Di Cesare S, Romiti ML, et al. Humoral immune responses and CD27+ B cells in children with DiGeorge syndrome (22q11.2 deletion syndrome). Pediatr Allergy Immunol 2006;17:382-8.

27. Waldmann H. Interactions between T and B cells: a review. J R Soc Med 1979;72:198-202.

28. Kobrynski LJ, Sullivan KE. Velocardiofacial syndrome, DiGeorge syndrome: the chromosome 22q11.2 deletion syndromes. Lancet 2007;370: 1443-52.

29. Tomita-Mitchell A, Mahnke DK, Larson JM, et al. Multiplexed quantitative real-time PCR to detect 22q11.2 deletion in patients with congenital heart disease. Physiol Genomics 2010;42A:52-60.
30. Vogel BH, Bonagura V, Weinberg GA, et al. Newborn screening for SCID in New York State: experience from the first two years. J Clin Immunol 2014;34:289-303.

31. Lingman Framme J, Borte S, von Döbeln U, Hammarström L, Oskarsdóttir S. Retrospective analysis of TREC based newborn screening results and clinical phenotypes in infants with the 22q11 deletion syndrome. J Clin Immunol 2014;34:514-9.

32. Gothelf D, Presburger G, Levy D, et al. Genetic, developmental, and physical factors associated with attention deficit hyperactivity disorder in patients with velocardiofacial syndrome. Am J Med Genet B Neuropsychiatr Genet 2004;126B:116-21.

33. Gothelf D, Schneider M, Green T, et al. Risk factors and the evolution of psychosis in 22q11.2 deletion syndrome: a longitudinal 2-site study. J Am Acad Child Adolesc Psychiatry 2013;52:1192-1203.e3.

34. Hazenberg MD, Verschuren MC, Hamann D, Miedema F, van Dongen JJ. T cell receptor excision circles as markers for recent thymic emigrants: basic aspects, technical approach, and guidelines for interpretation. J Mol Med (Berl) 2001;79:631-40.

35. Lev A, Simon AJ, Broides A, et al. Thymic function in MHC class IIdeficient patients. J Allergy Clin Immunol 2013;131:831-9. 